\title{
Explicit Euclidean Norm, Eigenvalues, Spectral Norm and Determinant of Circulant Matrix with the Generalized Tribonacci Numbers
}

\begin{abstract}
Yüksel Soykan
Department of Mathematics, Art and Science Faculty,

Zonguldak Bülent Ecevit University, 67100, Zonguldak, Turkey

e-mail: yuksel_soykan@hotmail.com

Abstract

In this paper, we obtain explicit Euclidean norm, eigenvalues, spectral norm and determinant of circulant matrix with the generalized Tribonacci (generalized $(r, s, t))$ numbers. We also present the sum of entries, the maximum column sum matrix norm and the maximum row sum matrix norm of this circulant matrix. Moreover, we give some bounds for the spectral norms of Kronecker and Hadamard products of circulant matrices of $(r, s, t)$ and Lucas $(r, s, t)$ numbers.
\end{abstract}

\section{Introduction}

The generalized $(r, s, t)$ sequence (or generalized Tribonacci sequence or generalized 3-step Fibonacci sequence)

$$
\left\{W_{n}\left(W_{0}, W_{1}, W_{2} ; r, s, t\right)\right\}_{n \geq 0}
$$

(or shortly $\left\{W_{n}\right\}_{n \geq 0}$ ) is defined as follows:

$$
W_{n}=r W_{n-1}+s W_{n-2}+t W_{n-3}, \quad W_{0}=a, W_{1}=b, W_{2}=c, \quad n \geq 3
$$

where $W_{0}, W_{1}, W_{2}$ are arbitrary complex (or real) numbers and $r, s, t$ are real numbers.

Received: December 27, 2020; Accepted: February 3, 2021

2020 Mathematics Subject Classification: 11B39, 11B83, 15A18, 15A60, 15B36, 11C20.

Keywords and phrases: $\quad(r, s, t)$ numbers, circulant matrix, Tribonacci numbers, norm, determinant, eigenvalues. 
This sequence has been studied by many authors, see for example 2, 3, 4, 10 , 11, $18,22,28,30,44,41,45,51,52$.

The sequence $\left\{W_{n}\right\}_{n \geq 0}$ can be extended to negative subscripts by defining

$$
W_{-n}=-\frac{s}{t} W_{-(n-1)}-\frac{r}{t} W_{-(n-2)}+\frac{1}{t} W_{-(n-3)}
$$

for $n=1,2,3, \ldots$ when $t \neq 0$. Therefore, recurrence 1 .1 holds for all integer $n$.

As $\left\{W_{n}\right\}$ is a third-order recurrence sequence (difference equation), its characteristic equation is

$$
x^{3}-r x^{2}-s x-t=0
$$

whose roots $\alpha, \beta, \gamma$ satisfy the following identities:

$$
\begin{aligned}
\alpha+\beta+\gamma & =r, \\
\alpha \beta+\alpha \gamma+\beta \gamma & =-s, \\
\alpha \beta \gamma & =t .
\end{aligned}
$$

It is well known that the generalized $(r, s, t)$ numbers (the generalized Tribonacci numbers) can be expressed, for all integers $n$, using Binet's formula

$$
W_{n}=\frac{p_{1} \alpha^{n}}{(\alpha-\beta)(\alpha-\gamma)}+\frac{p_{2} \beta^{n}}{(\beta-\alpha)(\beta-\gamma)}+\frac{p_{3} \gamma^{n}}{(\gamma-\alpha)(\gamma-\beta)},
$$

where

$$
\begin{gathered}
p_{1}=W_{2}-(\beta+\gamma) W_{1}+\beta \gamma W_{0}, p_{2}=W_{2}-(\alpha+\gamma) W_{1}+\alpha \gamma W_{0}, \\
p_{3}=W_{2}-(\alpha+\beta) W_{1}+\alpha \beta W_{0} .
\end{gathered}
$$

We need the special cases of the generalized $(r, s, t)$ sequence $\left\{W_{n}\right\}$ which are called $(r, s, t)$ and Lucas $(r, s, t)$ sequences. $(r, s, t)$ sequence $\left\{G_{n}\right\}_{n \geq 0}$ and Lucas $(r, s, t)$ sequence $\left\{H_{n}\right\}_{n \geq 0}$ are defined, respectively, by the third-order recurrence relations

$$
\begin{array}{ll}
G_{n+3}=r G_{n+2}+s G_{n+1}+t G_{n}, & G_{0}=0, G_{1}=1, G_{2}=r \\
H_{n+3}=r H_{n+2}+s H_{n+1}+t H_{n}, & H_{0}=3, H_{1}=r, H_{2}=2 s+r^{2}
\end{array}
$$


The sequences $\left\{G_{n}\right\}_{n \geq 0}$ and $\left\{H_{n}\right\}_{n \geq 0}$ can be extended to negative subscripts by defining

$$
\begin{aligned}
G_{-n} & =-\frac{s}{t} G_{-(n-1)}-\frac{r}{t} G_{-(n-2)}+\frac{1}{t} G_{-(n-3)}, \\
H_{-n} & =-\frac{s}{t} H_{-(n-1)}-\frac{r}{t} H_{-(n-2)}+\frac{1}{t} H_{-(n-3)}
\end{aligned}
$$

for $n=1,2,3, \ldots$ respectively. Therefore, recurrences (1.4) and 1.5 hold for all integers $n$.

Some special cases of $(r, s, t)$ sequence $\left\{G_{n}(0,1, r ; r, s, t)\right\}_{n \geq 0}$ and Lucas $(r, s, t)$ sequence $\left\{H_{n}\left(3, r, 2 s+r^{2} ; r, s, t\right)\right\}_{n \geq 0}$ are as follows:

1. $G_{n}(0,1,1 ; 1,1,1)=T_{n}$, Tribonacci sequence,

2. $H_{n}(3,1,3 ; 1,1,1)=K_{n}$, Tribonacci-Lucas sequence,

3. $G_{n}(0,1,2 ; 2,1,1)=P_{n}$, third order Pell sequence,

4. $H_{n}(3,2,6 ; 2,1,1)=Q_{n}$, third order Pell-Lucas sequence,

5. $G_{n}(0,1,0 ; 0,1,1)=U_{n}$, adjusted Padovan sequence,

6. $H_{n}(3,0,2 ; 0,1,1)=E_{n}$, Perrin (Padovan-Lucas) sequence.

Note that we use the Lucas $(r, s, t)$ numbers in Lemma 2.13 for calculating the determinants.

The following Theorem presents a summing formula of generalized Tribonacci numbers with positive subscripts.

Theorem 1.1. Let $x$ be a real or complex number. For $n \geq 0$, we have the following formula: If $t x^{3}+s x^{2}+r x-1 \neq 0$, then

$$
\sum_{k=0}^{n} x^{k} W_{k}=\frac{\Theta_{1}(x)}{\Theta(x)},
$$

where

$$
\begin{aligned}
\Theta_{1}(x)= & x^{n+3} W_{n+3}-(r x-1) x^{n+2} W_{n+2}-\left(s x^{2}+r x-1\right) x^{n+1} W_{n+1} \\
& -x^{2} W_{2}+x(r x-1) W_{1}+\left(s x^{2}+r x-1\right) W_{0}, \\
\Theta(x)= & t x^{3}+s x^{2}+r x-1 .
\end{aligned}
$$


Proof. It is given in 40].

Now, we give an alternative proof. By using Binet's formula of generalized Tribonacci numbers and the following identities

$$
\begin{aligned}
\alpha+\beta+\gamma & =r, \\
\alpha \beta+\alpha \gamma+\beta \gamma & =-s, \\
\alpha \beta \gamma & =t,
\end{aligned}
$$

we obtain

$$
\begin{aligned}
& \sum_{k=0}^{n-1} x^{k} W_{k}= \sum_{k=0}^{n-1} x^{k}\left(\frac{p_{1} \alpha^{k}}{(\alpha-\beta)(\alpha-\gamma)}+\frac{p_{2} \beta^{k}}{(\beta-\alpha)(\beta-\gamma)}+\frac{p_{3} \gamma^{k}}{(\gamma-\alpha)(\gamma-\beta)}\right) \\
&= \frac{p_{1}}{(\alpha-\beta)(\alpha-\gamma)}\left(\frac{(\alpha x)^{n}-1}{\alpha x-1}\right)+\frac{p_{2}}{(\beta-\alpha)(\beta-\gamma)}\left(\frac{(\beta x)^{n}-1}{\beta x-1}\right) \\
&+\frac{p_{3}}{(\gamma-\alpha)(\gamma-\beta)}\left(\frac{(\gamma x)^{n}-1}{\gamma x-1}\right) \\
&= \frac{p_{1}\left((\alpha x)^{n}-1\right)(\beta x-1)(\gamma x-1)(\beta-\gamma)}{(\alpha-\beta)(\alpha-\gamma)(\beta-\gamma)(\alpha x-1)(\beta x-1)(\gamma x-1)} \\
&= \frac{-p_{2}\left((\beta x)^{n}-1\right)(\alpha x-1)(\gamma x-1)(\gamma-\alpha)}{x^{n+2} W_{n+2}-x^{n+1}(r x-1) W_{n+1}-x^{n}\left(s x^{2}+r x-1\right) W_{n}} \\
& t x^{3}+s x^{2}+r x-1
\end{aligned} .
$$

Now, from the equality

$$
\sum_{k=0}^{n} x^{k} W_{k}=x^{n} W_{n}+\sum_{k=0}^{n-1} x^{k} W_{k},
$$

the claim of the theorem follows.

Note that

$$
\sum_{k=0}^{n-1} x^{k} W_{k}=\frac{\partial}{t x^{3}+s x^{2}+r x-1}
$$

where 


$$
\begin{gathered}
\partial=x^{n+2} W_{n+2}-x^{n+1}(r x-1) W_{n+1}-x^{n}\left(s x^{2}+r x-1\right) W_{n}-x^{2} W_{2} \\
+x(r x-1) W_{1}+\left(s x^{2}+r x-1\right) W_{0} .
\end{gathered}
$$

The case $x=1$ of the above theorem can be given as follows.

Theorem 1.2. For $n \geq 0$, we have the following formula: If $t+s+r-1 \neq 0$, then

$$
\sum_{k=0}^{n} W_{k}=\frac{\Theta_{1}}{\Theta},
$$

where

$$
\begin{aligned}
& \Theta_{1}=W_{n+3}-(r-1) W_{n+2}-(s+r-1) W_{n+1}-W_{2}+(r-1) W_{1}+(s+r-1) W_{0}, \\
& \Theta=t+s+r-1 .
\end{aligned}
$$

Let

$$
\Delta=\left(s+r t-t^{2}+1\right)(r+s+t-1)(r-s+t+1) .
$$

Theorem 1.3. If $\Delta \neq 0$, then

$$
\sum_{k=1}^{n} W_{k}^{2}=\frac{\Delta_{1}}{\Delta},
$$

where

$$
\begin{aligned}
\Delta_{1}= & -\left(t^{2}+r t+s-1\right) W_{n+3}^{2}-\left(r^{3} t+r^{2} t^{2}+r^{2} s+r^{2}+t^{2}+2 r s t+r t+s-1\right) W_{n+2}^{2} \\
& -\left(r^{3} t+r^{2} t^{2}+s^{2} t^{2}-r s^{2} t-s^{3}+r^{2} s+4 r s t+r^{2}+s^{2}+t^{2}+r t+s-1\right) W_{n+1}^{2} \\
& +2(r+t)(s+r t) W_{n+3} W_{n+2}+2 t(r+s t) W_{n+3} W_{n+1}-2 t(s-1)(s+r t) W_{n+2} W_{n+1} \\
& +\left(2 r s t+2 r^{2}+t^{2}+r t+s-1\right) W_{3}^{2}+\left(r^{3} t+r^{2} t^{2}+r^{2} s+2 r s t+r^{2}+t^{2}+r t+s-1\right) W_{2}^{2} \\
& +\left(r^{3} t+r^{2} t^{2}+s^{2} t^{2}-r s^{2} t-s^{3}+r^{2} s+4 r s t+r^{2}+s^{2}+t^{2}+r t+s-1\right) W_{1}^{2} \\
& -2(r+s t) W_{4} W_{3}-2 t\left(r^{2}-s^{2}+r t+s\right) W_{3} W_{2}+2 t(s-1)(s+r t) W_{2} W_{1} .
\end{aligned}
$$

Proof. It is given in Soykan [42, Theorem 2.1]. See also Soykan 43, Theorem 3.1].

Remark 1.4. Using Theorems 1.1, 1.2 and 1.3, we give relatively short proofs for our results which are given in the next section. 


\section{Main Results}

In this section, we obtain explicit Euclidean norm, eigenvalues, spectral norm and determinant of circulant matrix with the generalized Tribonacci (generalized $(r, s, t))$ numbers. We also present the sum of entries, the maximum column sum matrix norm and the maximum row sum matrix norm of this circulant matrix. Moreover, we give some bounds for the spectral norms of Kronecker and Hadamard products of circulant matrices of $(r, s, t)$ and Lucas $(r, s, t)$ numbers. For our work, we need to recall a $n \times n$ circulant matrix and various norm on matrices and their properties.

Let $n \geq 2$ be an integer. An $n \times n$ matrix $C=\left(c_{i j}\right) \in M_{n \times n}(\mathbb{C})$ is called a circulant matrix if it is of the form

$$
C=\left(\begin{array}{cccccc}
c_{0} & c_{1} & c_{2} & \cdots & c_{n-2} & c_{n-1} \\
c_{n-1} & c_{0} & c_{1} & \cdots & c_{n-3} & c_{n-2} \\
c_{n-2} & c_{n-1} & c_{0} & \cdots & c_{n-4} & c_{n-3} \\
\vdots & \vdots & \vdots & & \vdots & \vdots \\
c_{1} & c_{2} & c_{3} & \cdots & c_{n-1} & c_{0}
\end{array}\right)_{n \times n}
$$

i.e.

$$
c_{i j}=\left\{\begin{array}{cc}
c_{j-i} & , \quad j \geq i \\
c_{n+j-i} & , \quad j<i
\end{array}\right.
$$

and the circulant matrix $C$ is denoted by $C=\operatorname{Circ}\left(c_{0}, c_{1}, \ldots, c_{n-1}\right)$. Circulant matrix was first proposed by Davis in [7. This matrix has many interesting properties, and it is one of the most important research subject in the field of the computational and pure mathematics (see for example references given in Table $1)$.

For a $m \times n$ matrix $A=\left(a_{i j}\right) \in M_{m \times n}(\mathbb{C})$, the spectral norm of $A$ is given by

$$
\|A\|_{2}=\left(\max _{1 \leq i \leq n}\left|\lambda_{i}\left(A^{*} A\right)\right|\right)^{1 / 2}
$$

where $\lambda_{i}\left(A^{*} A\right)$ 's are the eigenvalues of the matrix $A^{*} A$ and $A^{*}$ is the conjugate of transpose of the matrix $A$. The Frobenius (or Euclidean) norm a $m \times n$ matrix 
$A=\left(a_{i j}\right)_{m \times n} \in M_{m \times n}(\mathbb{C})$ is as follows:

$$
\|A\|_{F}=\left(\sum_{i=1}^{m} \sum_{j=1}^{n}\left|a_{i j}\right|^{2}\right)^{1 / 2} .
$$

The following inequality holds for any matrix $A=\left(a_{i j}\right)_{m \times n} \in M_{n \times n}(\mathbb{C})$ (see 53 . Theorem 1 and Table 1]):

$$
\frac{1}{\sqrt{n}}\|A\|_{F} \leq\|A\|_{2} \leq\|A\|_{F} .
$$

It follows that

$$
\|A\|_{2} \leq\|A\|_{F} \leq \sqrt{n}\|A\|_{2} .
$$

In literature there are other types of norms of matrices. The maximum column sum matrix norm of $n \times n$ matrix $A=\left(a_{i j}\right)$ is $\|A\|_{1}=\max _{1 \leq j \leq n} \sum_{i=1}^{n}\left|a_{i j}\right|$ and the maximum row sum matrix norm is $\|A\|_{\infty}=\max _{1 \leq i \leq n} \sum_{j=1}^{n}\left|a_{i j}\right|$.

The maximum column length norm $c_{1}($.$) and the maximum row length norm$ $r_{1}($.$) of an matrix of order m \times n$ are defined as follows:

$$
c_{1}(A)=\max _{1 \leq j \leq n}\left(\sum_{i=1}^{n}\left|a_{i j}\right|^{2}\right)^{1 / 2} \text { and } r_{1}(A)=\max _{1 \leq i \leq n}\left(\sum_{j=1}^{n}\left|a_{i j}\right|^{2}\right)^{1 / 2} .
$$

There is a relation between $\|\cdot\|_{2}, c_{1}($.$) and r_{1}($.$) norms:$

Lemma 2.1. 15] For any matrices $A=\left(a_{i j}\right)_{m \times n} \in M_{m \times n}(\mathbb{C})$ and $B=$ $\left(b_{i j}\right)_{m \times n} \in M_{m \times n}(\mathbb{C})$, we have

$$
\|A \circ B\|_{2} \leq r_{1}(A) c_{1}(B)
$$

and

$$
\|A \circ B\|_{2} \leq\|A\|_{2}\|B\|_{2}
$$

and

$$
\|A \otimes B\|_{2}=\|A\|_{2}\|B\|_{2}
$$


where $A \circ B$ is the Hadamard product which is defined by

$$
A \circ B=\left(a_{i j} b_{i j}\right)
$$

$A \otimes B$ is the Kronecker product which is defined by

$$
A \otimes B=\left(a_{i j} B\right)
$$

and $r_{1}(A)=\max _{1 \leq i \leq n}\left(\sum_{j=1}^{n}\left|a_{i j}\right|^{2}\right)^{1 / 2}, c_{1}(B)=\max _{1 \leq j \leq n}\left(\sum_{i=1}^{n}\left|b_{i j}\right|^{2}\right)^{1 / 2}$.

The Kronecker product is also sometimes called matrix direct product.

For more details on norm of matrices, see for example [14. In the following Table 1, we present a few special study on the Frobenius norm, spectral norm, maximum column length norm and maximum row length norm of circulant (r-circulant, geometric circulant, semicirculant) matrices with the generalized $m$-step Fibonacci sequences which require sum formulas of second powers of numbers in $m$-step Fibonacci sequences $(m=2,3,4)$. For $m$-step Fibonacci sequences, see, for example, 39].

\begin{tabular}{|c|c|}
\hline Name of sequence & Papers \\
\hline second order $\downarrow$ & second order $\downarrow$ \\
\hline Fibonacci, Lucas & 8, 9, $13,17,19,27,31,32,33,34,36,37,38,46$ \\
\hline Pell, Pell-Lucas & 1147 \\
\hline Jacobsthal, Jacobsthal-Lucas & $24,48,49,50$ \\
\hline third order $\downarrow$ & third order $\downarrow$ \\
\hline Tribonacci, Tribonacci-Lucas & $16,25,26$ \\
\hline Padovan, Perrin & $6.21,29$ \\
\hline fourth order $\downarrow$ & fourth order $\downarrow$ \\
\hline Tetranacci, Tetranacci-Lucas & 20 \\
\hline
\end{tabular}

Table 1. Papers on the norms.

See also Polatl 23 for the spectral norms of k-circulant matrices with a type of Catalan triangle numbers.

We need the following three lemmas for our calculations. 
Lemma 2.2. [7, 5] Let $C=\operatorname{Circ}\left(c_{0}, c_{1}, \ldots, c_{n-1}\right)$ be a $n \times n$ circulant matrix. Then the eigenvalues of $C$ are

$$
\lambda_{j}(C)=\sum_{k=0}^{n-1} \omega^{-j k} c_{k}
$$

and the corresponding eigenvectors are

$$
v_{j}=\left(1, \omega^{-j}, \omega^{-2 j}, \omega^{-3 j}, \ldots, \omega^{-(n-1) j}\right)^{T}
$$

where $\omega=\exp (2 \pi i / n), i=\sqrt{-1}, j=0,1,2, \ldots, n-1$.

Note that $(2.2)$ is equivalent to

$$
\lambda_{n-j}(C)=\sum_{p=0}^{n-1} \omega^{j p} c_{p}, \quad j=0,1,2, \ldots, n-1
$$

or

$$
\lambda_{m}(C)=\sum_{p=0}^{n-1} \omega^{m p} c_{p}, \quad m=n, n-1, \ldots, 2,1
$$

or

$$
\mu_{j}(C)=\sum_{p=0}^{n-1} \omega^{j p} c_{p}, \quad j=0,1, \ldots, n-1 \quad\left(\mu_{j}=\lambda_{n-j}\right) .
$$

We have the determinants and inverses of nonsingular circulant matrices 35 . 12, 7, 5:

Lemma 2.3. Determinant of a circulant matrix $C$ is

$$
\operatorname{det}(C)=\prod_{j=0}^{n-1} \lambda_{j}(C)=\prod_{j=0}^{n-1}\left(\sum_{p=0}^{n-1} \omega^{-j p} c_{p}\right)=\prod_{j=0}^{n-1}\left(\sum_{p=0}^{n-1} \omega^{j p} c_{p}\right)
$$

and if $C$ is nonsingular circulant matrix then its inverse is

$$
C^{-1}=\operatorname{Circ}\left(a_{0}, a_{1}, \ldots, a_{n-1}\right)
$$

where

$$
a_{n-p}=\frac{1}{n} \sum_{j=0}^{n-1}\left(\sum_{k=0}^{n-1} \omega^{-j k} W_{k}\right)^{-1} \omega^{-p j}=\frac{1}{n} \sum_{j=0}^{n-1} \lambda_{j}^{-1} \omega^{-p j}, \quad p=1,2,3, \ldots, n
$$


or

$$
a_{p}=\frac{1}{n} \sum_{j=0}^{n-1}\left(\sum_{k=0}^{n-1} \omega^{j k} W_{k}\right)^{-1} \omega^{-p j}, \quad p=0,1,2, \ldots, n-1 .
$$

Lemma 2.4. 14 Let $A$ be a $n \times n$ matrix with eigenvalues $\lambda_{1}, \lambda_{2}, \lambda_{3}, \ldots, \lambda_{n}$. Then, $A$ is a normal matrix if and only if the eigenvalues of $A A^{*}$ are $\left|\lambda_{1}\right|^{2},\left|\lambda_{2}\right|^{2},\left|\lambda_{3}\right|^{2}, \ldots,\left|\lambda_{n}\right|^{2}$ where $A^{*}$ is the conjugate of transpose of the matrix A.

Next, we define circulant matrix with generalized $(r, s, t)$ numbers entries.

Definition 2.5. A $n \times n$ circulant matrix with generalized $(r, s, t)$ (generalized Tribonacci) numbers entries is defined by

$$
C_{n}(W)=\left(\begin{array}{cccccc}
W_{0} & W_{1} & W_{2} & \cdots & W_{n-2} & W_{n-1} \\
W_{n-1} & W_{0} & W_{1} & \cdots & W_{n-3} & W_{n-2} \\
W_{n-2} & W_{n-1} & W_{0} & \cdots & W_{n-4} & W_{n-3} \\
\vdots & \vdots & \vdots & & \vdots & \vdots \\
W_{1} & W_{2} & W_{3} & \cdots & W_{n-1} & W_{0}
\end{array}\right)_{n \times n}=\operatorname{Circ}\left(W_{0}, W_{1}, \ldots, W_{n-1}\right)
$$

We call this matrix as generalized Tribonacci (generalized $(r, s, t)$ ) circulant matrix.

We consider two special cases of generalized Tribonacci (generalized $(r, s, t))$ circulant matrix, namely $(r, s, t))$ circulant matrix: $C_{n}(G)=$ $\operatorname{Circ}\left(G_{0}, G_{1}, \ldots, G_{n-1}\right)$ and Lucas $(r, s, t)$ circulant matrix: $C_{n}(H)=$ $\operatorname{Circ}\left(H_{0}, H_{1}, \ldots, H_{n-1}\right)$.

We denote the sum of entries of $C_{n}(W)$ as $S\left(C_{n}(W)\right)$.

Lemma 2.6. The sum of entries of $C_{n}(W)$ is

$$
S\left(C_{n}(W)\right)=n\left(-W_{n}+\frac{\Theta_{1}}{\Theta}\right)
$$

where $\Theta_{1}$ and $\Theta$ are as in Theorem 1.2. 
Proof. From the definition of $C_{n}(W)$, using Theorem 1.2, we obtain

$$
S\left(C_{n}(W)\right)=n \sum_{i=0}^{n-1} W_{i}=n\left(-W_{n}+\sum_{i=0}^{n} W_{i}\right)=n\left(-W_{n}+\frac{\Theta_{1}}{\Theta}\right) .
$$

Next, we present the maximum column sum matrix norm $\left\|C_{n}(W)\right\|_{1}$ and the maximum row sum matrix norm $\left\|C_{n}(W)\right\|_{\infty}$ of matrix $C_{n}(W)=\left(a_{i j}\right)$ under certain condition on the generalized Tribonaci sequence $W_{n}$.

Theorem 2.7. Suppose that $W_{p} \geq 0$ for all the nonnegative integers $p$. Then we have the following formula:

$$
\left\|C_{n}(W)\right\|_{1}=\left\|C_{n}(W)\right\|_{\infty}=-W_{n}+\frac{\Theta_{1}}{\Theta} .
$$

Proof. From the definition of the matrix $C_{n}(W)=\left(a_{i j}\right)$ we can write

$$
\begin{aligned}
\left\|C_{n}(W)\right\|_{1} & =\max _{1 \leq j \leq n} \sum_{i=1}^{n}\left|a_{i j}\right|=\max _{1 \leq j \leq n}\left\{\left|a_{1 j}\right|+\left|a_{2 j}\right|+\left|a_{3 j}\right|+\ldots+\left|a_{n j}\right|\right\} \\
& =\left|a_{1 n}\right|+\left|a_{2 n}\right|+\left|a_{3 n}\right|+\ldots+\left|a_{n n}\right| \\
& =W_{n-1}+W_{n-2}+\ldots+W_{3}+W_{2}+W_{1}+W_{0} \\
& =-W_{n}+\sum_{i=0}^{n} W_{i} \\
& =-W_{n}+\frac{\Theta_{1}}{\Theta}
\end{aligned}
$$

Similarly, we have

$$
\left\|C_{n}(W)_{k}\right\|_{\infty}=-W_{n}+\frac{\Theta_{1}}{\Theta}
$$

The following theorem gives the Euclidean (Frobenius) norm of circulant matrix $C_{n}(W)$. 
Theorem 2.8. The Euclidean norm of circulant matrix $C_{n}(W)$ is:

$$
\left\|C_{n}(W)\right\|_{F}=\sqrt{n\left(W_{0}^{2}-W_{n}^{2}+\frac{\Delta_{1}}{\Delta}\right)}
$$

where $\Delta$ and $\Delta_{1}$ are as in Theorem 1.3.

Proof. From the definition of the Euclidean norm of a matrix, using Theorem 1.3 . we obtain

$$
\left(\left\|C_{n}(W)\right\|_{F}\right)^{2}=n \sum_{i=0}^{n-1} W_{i}^{2}=n\left(W_{0}^{2}-W_{n}^{2}+\sum_{i=1}^{n} W_{i}^{2}\right)=n\left(W_{0}^{2}-W_{n}^{2}+\frac{\Delta_{1}}{\Delta}\right) .
$$

It follows that

$$
\left\|C_{n}(W)\right\|_{F}=\sqrt{n\left(W_{0}^{2}-W_{n}^{2}+\frac{\Delta_{1}}{\Delta}\right)} .
$$

The following theorem gives us the eigenvalues of the matrix in (2.3).

Theorem 2.9. The eigenvalues of $C_{n}(W)$ are

$$
\lambda_{j}\left(C_{n}(W)\right)=\frac{\Phi_{j}}{r \omega^{-j}+s \omega^{-2 j}+t \omega^{-3 j}-1}
$$

where $\Phi_{j}=\left(W_{n}-W_{0}\right)-\left(-W_{n+1}+r W_{n}+W_{1}-r W_{0}\right) \omega^{-j}+\left(W_{n+2}-r W_{n+1}-\right.$ $\left.s W_{n}-W_{2}+r W_{1}+s W_{0}\right) \omega^{-2 j}$, and

$$
\begin{aligned}
\omega & =\exp (2 \pi i / n), \\
j & =0,1,2,3, \ldots, n-1 .
\end{aligned}
$$

Proof. By using 1.6) or Theorem 1.1 (by putting $x=\omega^{-j}$ ), we obtain

$$
\begin{aligned}
& \lambda_{j}\left(C_{n}(W)\right) \\
= & \sum_{k=0}^{n-1} \omega^{-j k} W_{k} \\
= & -\omega^{-j n} W_{n}+\sum_{k=0}^{n}\left(\omega^{-j}\right)^{k} W_{k} \\
= & \frac{-\omega^{-2 j} W_{2}+\omega^{-j}\left(r \omega^{-j}-1\right) W_{1}+\left(s \omega^{-2 j}+r \omega^{-j}-1\right) W_{0}}{t \omega^{-3 j}+s \omega^{-2 j}+r \omega^{-j}-1} W_{n+2}-\omega^{-j(n+1)}\left(r \omega^{-j}-1\right) W_{n+1}-\omega^{-j n}\left(s \omega^{-2 j}+r \omega^{-j}-1\right) W_{n}
\end{aligned} .
$$


By simplifying the last equality, we get

$$
\lambda_{j}\left(C_{n}(W)\right)=\frac{\Phi_{j}}{r \omega^{-j}+s \omega^{-2 j}+t \omega^{-3 j}-1} .
$$

The following theorem presents the spectral norm of $C_{n}(W)$.

Theorem 2.10. Suppose that $W_{p} \geq 0$ for all the nonnegative integers $p$. The spectral norm of $C_{n}(W)$ is

$\left\|C_{n}(W)\right\|_{2}=\frac{W_{n+2}-(r-1) W_{n+1}-(s+r-1) W_{n}-W_{2}+(r-1) W_{1}+(s+r-1) W_{0}}{t+s+r-1}$.

Proof. For $j=0$, we have

$$
\lambda_{0}\left(C_{n}(W)\right)=\sum_{p=0}^{n-1} \omega^{-0 \times k} W_{k}=\sum_{p=0}^{n-1} W_{p} .
$$

Note that the matrix $C_{n}(W)$ is a normal matrix since

$$
C_{n}(W)^{*} C_{n}(W)=C_{n}(W) C_{n}(W)^{*} .
$$

Now, using Lemma 2.4, we see that

$$
\left\|C_{n}(W)\right\|_{2}=\left(\max _{1 \leq j \leq n} \mid \lambda_{j}\left(\left.C_{n}(W)\right|^{2}\right)^{1 / 2} .\right.
$$

In the last equality, if we take $j=0$, then $\lambda_{0}$ becomes the maximum eigenvalue because

$$
\left\|C_{n}(W)\right\|_{2}=\left(\max _{1 \leq j \leq n}\left|\lambda_{j}\left(C_{n}(W)\right)\right|^{2}\right)^{1 / 2}=\left(\left|\lambda_{0}\left(C_{n}(W)\right)\right|^{2}, \max _{1 \leq j \leq n-1}\left|\lambda_{j}\left(C_{n}(W)\right)\right|^{2}\right)^{1 / 2}
$$

and

$$
\max _{1 \leq j \leq n-1}\left|\lambda_{j}\left(C_{n}(W)\right)\right|=\left|\sum_{p=0}^{n-1} \omega^{-j p} W_{p}\right| \leq \sum_{p=0}^{n-1}\left|\omega^{-j p}\right|\left|W_{p}\right|=\sum_{k=0}^{n-1}\left|W_{p}\right|=\sum_{k=0}^{n-1} W_{p}=\lambda_{0}\left(C_{n}(W)\right) .
$$

Hence

$$
\left\|C_{n}(W)\right\|_{2}=\left|\lambda_{0}\left(C_{n}(W)\right)\right|=\sum_{p=0}^{n-1} W_{p}
$$


By applying Theorem 2.9 (or from Theorem 1.1), we obtain

$$
\begin{aligned}
& \left\|C_{n}(W)\right\|_{2} \\
= & \frac{W_{n+2}-(r-1) W_{n+1}-(s+r-1) W_{n}-W_{2}+(r-1) W_{1}+(s+r-1) W_{0}}{t+s+r-1} .
\end{aligned}
$$

The following corollary presents the spectral norms of $(r, s, t))$ circulant matrix: $C_{n}(G)=\operatorname{Circ}\left(G_{0}, G_{1}, \ldots, G_{n-1}\right)$ and Lucas $(r, s, t)$ circulant matrix: $C_{n}(H)=\operatorname{Circ}\left(H_{0}, H_{1}, \ldots, H_{n-1}\right)$.

Corollary 2.11. Suppose that $G_{p} \geq 0$ and $H_{p} \geq 0$ for all the nonnegative integers $p$. The spectral norm of $C_{n}(G)$ and $C_{n}(H)$ are

$$
\left\|C_{n}(G)\right\|_{2}=\frac{G_{n+2}-(r-1) G_{n+1}-(s+r-1) G_{n}-1}{t+s+r-1}
$$

and

$$
\left\|C_{n}(H)\right\|_{2}=\frac{H_{n+2}-(r-1) H_{n+1}-(s+r-1) H_{n}+2 r+s-3}{t+s+r-1}
$$

respectively.

Proof. Take $W_{n}=G_{n}, G_{0}=0, G_{1}=1, G_{2}=r$ and $W_{n}=H_{n}, H_{0}=3, H_{1}=$ $r, H_{2}=2 s+r^{2}$ in Theorem 2.10 .

The following corollary presents properties of the spectral norms of Hadamard product and Kronecker product of $C_{n}(G)=\operatorname{Circ}\left(G_{0}, G_{1}, \ldots, G_{n-1}\right)$ and $C_{n}(H)=$ $\operatorname{Circ}\left(H_{0}, H_{1}, \ldots, H_{n-1}\right)$

Corollary 2.12. Suppose that $G_{p} \geq 0$ and $H_{p} \geq 0$ for all the nonnegative integers $p$. The spectral norm of Hadamard product of $C_{n}(G)$ and $C_{n}(H)$ has the following property:

$$
\left\|C_{n}(G) \circ C_{n}(H)\right\|_{2} \leq \frac{\Upsilon}{(t+s+r-1)^{2}}
$$

and the spectral norm of Kronecker product of $C_{n}(G)$ and $C_{n}(H)$ has the following property:

$$
\left\|C_{n}(G) \otimes C_{n}(H)\right\|_{2}=\frac{\Upsilon}{(t+s+r-1)^{2}}
$$


where $\Upsilon=\left(G_{n+2}-(r-1) G_{n+1}-(s+r-1) G_{n}-1\right)\left(H_{n+2}-(r-1) H_{n+1}-(s+\right.$ $\left.r-1) H_{n}+2 r+s-3\right)$.

Proof. Since $\left\|C_{n}(G) \circ C_{n}(H)\right\|_{2} \leq\left\|C_{n}(G)\right\|_{2}\left\|C_{n}(H)\right\|_{2}$ and $\left\|C_{n}(G) \otimes C_{n}(H)\right\|_{2}=$ $\left\|C_{n}(G)\right\|_{2}\left\|C_{n}(H)\right\|_{2}$, the proof is trivial from Corollary 2.11

Next, we present the determinant of $C_{n}(W)$.

Theorem 2.13. The determinant of $C_{n}(W)$ is given by

$$
\operatorname{det}\left(C_{n}(W)\right)=\frac{\Lambda_{1}^{n}\left(1-\left(\frac{\Lambda_{2}-\sqrt{\Lambda_{2}^{2}-4 \Lambda_{1} \Lambda_{3}}}{2 \Lambda_{1}}\right)^{n}-\left(\frac{\Lambda_{2}+\sqrt{\Lambda_{2}^{2}-4 \Lambda_{1} \Lambda_{3}}}{2 \Lambda_{1}}\right)^{n}+\left(\frac{\Lambda_{3}}{\Lambda_{1}}\right)^{n}\right)}{(-1)^{n+1}\left(H_{n}+\left(1-H_{-n}\right) t^{n}-1\right)}
$$

where

$$
\begin{aligned}
\omega & =\exp (2 \pi i / n), \\
\Lambda_{1} & =W_{n}-W_{0}, \\
\Lambda_{2} & =-W_{n+1}+r W_{n}+W_{1}-r W_{0}, \\
\Lambda_{3} & =W_{n+2}-r W_{n+1}-s W_{n}-W_{2}+r W_{1}+s W_{0},
\end{aligned}
$$

Proof. By considering identities

$$
\begin{aligned}
& \prod_{j=0}^{n-1}\left(x-y \omega^{-j}\right)=x^{n}-y^{n}, \\
& \prod_{j=0}^{n-1}\left(x-y \omega^{-j}+z \omega^{-2 j}\right) \\
= & x^{n}\left(1-\left(\frac{y-\sqrt{y^{2}-4 x z}}{2 x}\right)^{n}-\left(\frac{y+\sqrt{y^{2}-4 x z}}{2 x}\right)^{n}+\left(\frac{z}{x}\right)^{n}\right)
\end{aligned}
$$

and

$$
\left(r \omega^{-j}+s \omega^{-2 j}+t \omega^{-3 j}-1\right)=\left(\alpha \omega^{-j}-1\right)\left(\beta \omega^{-j}-1\right)\left(\gamma \omega^{-j}-1\right),
$$

we see that

$$
\prod_{j=0}^{n-1}\left(r \omega^{-j}+s \omega^{-2 j}+t \omega^{-3 j}-1\right)=(-1)^{n+1}\left(H_{n}+\left(1-H_{-n}\right) t^{n}-1\right) .
$$


and

$$
\begin{aligned}
& \prod_{j=0}^{n-1}\left(\left(W_{n}-W_{0}\right)-\left(-W_{n+1}+r W_{n}+W_{1}-r W_{0}\right) \omega^{-j}\right. \\
\left.+\left(W_{n+2}-r W_{n+1}-s W_{n}-W_{2}+r W_{1}+s W_{0}\right) \omega^{-2 j}\right) & \\
= & \Lambda_{1}^{n}\left(1-\left(\frac{\Lambda_{2}-\sqrt{\Lambda_{2}^{2}-4 \Lambda_{1} \Lambda_{3}}}{2 \Lambda_{1}}\right)^{n}-\left(\frac{\Lambda_{2}+\sqrt{\Lambda_{2}^{2}-4 \Lambda_{1} \Lambda_{3}}}{2 \Lambda_{1}}\right)^{n}+\left(\frac{\Lambda_{3}}{\Lambda_{1}}\right)^{n}\right) \\
\omega= & \exp (2 \pi i / n), \\
\Phi_{j}= & \left(W_{n}-W_{0}\right)-\left(-W_{n+1}+r W_{n}+W_{1}-r W_{0}\right) \omega^{-j} \\
& +\left(W_{n+2}-r W_{n+1}-s W_{n}-W_{2}+r W_{1}+s W_{0}\right) \omega^{-2 j}
\end{aligned}
$$

and

$$
\begin{aligned}
\Lambda_{1} & =W_{n}-W_{0}, \\
\Lambda_{2} & =-W_{n+1}+r W_{n}+W_{1}-r W_{0}, \\
\Lambda_{3} & =W_{n+2}-r W_{n+1}-s W_{n}-W_{2}+r W_{1}+s W_{0},
\end{aligned}
$$

From Theorem 2.10, we have

$$
\begin{aligned}
\operatorname{det}\left(C_{n}(W)\right) & =\prod_{j=0}^{n-1} \lambda_{j}\left(C_{n}(W)\right) \\
& =\prod_{j=0}^{n-1} \frac{\Phi_{j}}{\left(r \omega^{-j}+s \omega^{-2 j}+t \omega^{-3 j}-1\right)} \\
& =\frac{\prod_{j=0}^{n-1} \Phi_{j}}{\prod_{j=0}^{n-1}\left(r \omega^{-j}+s \omega^{-2 j}+t \omega^{-3 j}-1\right)} \\
& =\frac{\Lambda_{1}^{n}\left(1-\left(\frac{\Lambda_{2}-\sqrt{\Lambda_{2}^{2}-4 \Lambda_{1} \Lambda_{3}}}{2 \Lambda_{1}}\right)^{n}-\left(\frac{\Lambda_{2}+\sqrt{\Lambda_{2}^{2}-4 \Lambda_{1} \Lambda_{3}}}{2 \Lambda_{1}}\right)^{n}+\left(\frac{\Lambda_{3}}{\Lambda_{1}}\right)^{n}\right)}{(-1)^{n+1}\left(H_{n}+\left(1-H_{-n}\right) t^{n}-1\right)}
\end{aligned}
$$

which completes the proof.

Remark 2.14. Note that choosing suitable values on $r, s, t$ and $W_{0}=a, W_{1}=$ $b, W_{2}=c$ (initial values) in Theorems 2.8, 2.9, 2.10, 2.13, lower and upper bounds 
of the Euclidean norm, eigenvalues, the spectral norm and determinant of circulant matrices for the special case of all third order sequences can be obtained.

\section{References}

[1] E. Alptekin and G. Pell, Pell-Lucas ve Modified Pell sayıları ile tanımlı circulant ve semicirculant matrisler, Doctoral dissertation, Selçuk Üniversitesi Fen Bilimleri Enstitüsü, 2005.

[2] I. Bruce, A modified Tribonacci sequence, Fibonacci Quart. 22(3) (1984), 244-246.

[3] M. Catalani, Identities for Tribonacci-related sequences, 2012. arXiv:math/0209179

[4] E. Choi, Modular Tribonacci numbers by matrix method, J. Korean Soc. Math. Educ. Ser. B Pure Appl. Math. 20(3) (2013), 207-221. https://doi.org/10.7468/jksmeb.2013.20.3.207

[5] R.E. Cline, R.J. Plemmons and G. Worm, Generalized inverses of certain Toeplitz matrices, Linear Algebra Appl. 8 (1974), 25-33. https://doi.org/10.1016/0024-3795(74)90004-4

[6] A. Coskun and N. Taskara, On the some properties of circulant matrices with third order linear recurrent sequences, Math. Sci. Appl. E-Notes 6(1) (2018), 12-18. https://doi.org/10.36753/mathenot.421748

[7] P.J. Davis, Circulant Matrices, John Wiley \& Sons, New York, 1979.

[8] O. Deveci, E. Karaduman and C.M. Campbell, The Fibonacci-circulant sequences and their applications, Iran. J. Sci. Technol. Trans. A Sci. 41(4) (2017), 1033-1038. https://doi.org/10.1007/s40995-017-0317-7

[9] O. Deveci, On The Fibonacci-circulant p-sequences, Util. Math. 108 (2018), 107-124.

[10] M. Elia, Derived sequences, the Tribonacci recurrence and cubic forms, Fibonacci Quart. 39(2) (2001), 107-115.

[11] M.C. Er, Sums of Fibonacci numbers by matrix methods, Fibonacci Quart. 22(3) (1984), 204-207. 
[12] I.J. Good, On the inversion of circulant matrices, Biometrica 37 (1950), 185-186. https://doi.org/10.1093/biomet/37.1-2.185

[13] C. He, J. Ma, K. Zhang and Z. Wang, The upper bound estimation on the spectral norm of r-circulant matrices with the Fibonacci and Lucas numbers, J. Inequal. Appl. 2015:72, 2015. https://doi.org/10.1186/s13660-015-0596-5

[14] R.A. Horn and C.R. Johnson, Matrix Analysis, 2nd ed., Cambridge University Press, Cambridge, 2012.

[15] R.A. Horn and C.R. Johnson, Topics in Matrix Analysis, Cambridge University Press, Cambridge, 1991.

[16] C. Kizılates and N. Tuglu, On the norms of geometric and symmetric geometric circulant matrices with the Tribonacci number, Gazi University Journal of Science $31(2)$ (2018), 555-567.

[17] C. Kizlates and N. Tuglu, On the bounds for the spectral norms of geometric circulant matrices, J. Inequal. Appl. 2016(1), Article ID 312, 2016. https://doi.org/10.1186/s13660-016-1255-1

[18] P.Y. Lin, De Moivre-Type identities for the Tribonacci numbers, Fibonacci Quart. 26 (1988), 131-134.

[19] J.K. Merikoski, P. Haukkanen, M. Mattila and T. Tossavainen, On the spectral and Frobenius norm of a generalized Fibonacci r-circulant matrix, Spec. Matrices 6 (2018), 23-36. https://doi.org/10.1515/spma-2018-0003

[20] A. Özkoç and E. Ardıyok, Circulant and negacyclic matrices via tetranacci numbers, Honam Math. J. 38(4) (2016), 725-738.

https://doi.org/10.5831/HMJ.2016.38.4.725

[21] A. Pacheenburawana and W. Sintunavarat, On the spectral norms of $r$-circulant matrices with the Padovan and Perrin sequences, J. Math. Anal. 9(3) (2018), $110-122$.

[22] S. Pethe, Some identities for Tribonacci sequences, Fibonacci Quart. 26(2) (1988), 144-151.

[23] E. Polatll, On the bounds for the spectral norms of $r$-circulant matrices with a type of Catalan triangle numbers, Journal of Science and Arts 3(48) (2019), 575-578. 
[24] B. Radicic, On $k$-circulant matrices involving the Jacobsthal numbers, Revista de la Union Matematica Argentina 60(2) (2019), 431-442. https://doi.org/10.33044/revuma.v60n2a10

[25] Z. Raza, M. Riaz and M.A. Ali, Some inequalities on the norms of special matrices with generalized Tribonacci and Generalized Pell-Padovan sequences, 2015. arXiv, http://arxiv.org/abs/1407.1369v2

[26] Z. Raza and M.A. Ali, On the norms of circulant, $r$-circulant, semi-circulant and Hankel matrices with Tribonacci sequence, 2014. arxiv, http://arxiv.org/abs/1407.1369v1

[27] Z. Raza and M.A. Ali, On the norms of some special matrices with generalized Fibonacci sequence, J. Appl. Math. Inform. 33(5-6) (2015), 593-605.

https://doi.org/10.14317/jami.2015.593

[28] A. Scott, T. Delaney and V. Hoggatt, Jr., The Tribonacci sequence, Fibonacci Quart. 15(3) (1977), 193-200.

[29] W. Sintunavarat, The upper bound estimation for the spectral norm of $r$-circulant and symmetric $r$-circulant matrices with the Padovan sequence, J. Nonlinear Sci. Appl. 9 (2016), 92-101. https://doi.org/10.22436/jnsa.009.01.09

[30] A. Shannon, Tribonacci numbers and Pascal's pyramid, Fibonacci Quart. 15(3) (1977), pp. 268 and 275, 1977.

[31] S. Shen and J. Cen, On the norms of circulant matrices with the $(k, h)$-Fibonacci and $(k, h)$-Lucas numbers, Int. J. Contemp. Math. Sci. 6(18) (2011), 887-894.

[32] S. Shen, The spectral norms of circulant matrices involving $(k, h)$-Fibonacci and (k,h)-Lucas numbers, Int. J. Contemp. Math. Sci. 9(14) (2014), 661-665. https://doi.org/10.12988/ijcms.2014.4996

[33] S. Shen and J. Cen, On the spectral norms of $r$-circulant matrices with the $k$-Fibonacci and $k$-Lucas numbers, Int. J. Contemp. Math. Sci. 5(12) (2010), 569-578.

[34] S. Shen and J. Cen, On the bounds for the norms of $r$-circulant matrices with the Fibonacci and Lucas numbers, Appl. Math. Comput. 216 (2010), 2891-2897. https://doi.org/10.1016/j.amc.2010.03.140 
[35] S. Shen, J. Cen and Y. Hao, On the determinants and inverses of circulant matrices with Fibonacci and Lucas numbers, Appl. Math. Comput. 217 (2011), 9790-9797. https://doi.org/10.1016/j.amc.2011.04.072

[36] B. Shi, The spectral norms of geometric circulant matrices with the generalized $k$-Horadam numbers, J. Inequal. Appl., 2018:14, 2018. https://doi.org/10.1186/s13660-017-1608-4

[37] S. Solak, On the norms of circulant matrices with the Fibonacci and Lucas numbers, Appl. Math. Comput. 160 (2005), 125-132. https://doi.org/10.1016/j.amc.2003.08.126

[38] S. Solak, Erratum to "On the norms of circulant matrices with the Fibonacci and Lucas numbers" [Appl. Math. Comput. 160 (2005), 125-132], Appl. Math. Comput. 190 (2007), 1855-1856. https://doi.org/10.1016/j.amc.2007.02.075

[39] Y. Soykan, Simson identity of generalized m-step Fibonacci numbers, Int. J. Adv. Appl. Math. Mech. 7(2) (2019), 45-56.

[40] Y. Soykan, Generalized Tribonacci numbers: summing formulas, Int. J. Adv. Appl. Math. Mech. 7(3) (2020), 57-76.

[41] Y. Soykan, A study on generalized $(r, s, t)$-numbers, MathLAB J. 7 (2020), 101-129.

[42] Y. Soykan, A closed formula for the sums of squares of generalized Tribonacci numbers, Journal of Progressive Research in Mathematics 16(2) (2020), 2932-2941.

[43] Y. Soykan, On the sums of squares of generalized Tribonacci numbers: closed formulas of $\sum_{k=0}^{n} x^{k} W_{k}^{2}$, Archives of Current Research International 20(4) (2020), 22-47. https://doi.org/10.9734/acri/2020/v20i430187

[44] Y. Soykan, Tribonacci and Tribonacci-Lucas sedenions, Mathematics 7(1) (2019), 74. https://doi.org/10.3390/math7010074

[45] W. Spickerman, Binet's formula for the Tribonacci sequence, Fibonacci Quart. 20 (1982), 118-120.

[46] N. Tuglu and C. Kizılates, On the norms of circulant and $r$-circulant matrices with the hyperharmonic Fibonacci numbers, J. Inequal. Appl. 2015, Article ID 253, 2015. https://doi.org/10.1186/s13660-015-0778-1 
[47] R. Türkmen and H. Gökbaş, On the spectral norm of $r$-circulant matrices with the Pell and Pell-Lucas numbers, J. Inequal. Appl. 2016:65, 2016. https://doi.org/10.1186/s13660-016-0997-0

[48] Ş. Uygun, Some bounds for the norms of circulant matrices with the $k$-Jacobsthal and $k$-Jacobsthal Lucas numbers, Journal of Mathematics Research 8(6) (2016), 133-138. https://doi.org/10.5539/jmr.v8n6p133

[49] Ş. Uygun and S. Yaşamalı, On the bounds for the norms of circulant matrices with the Jacobsthal and Jacobsthal-Lucas numbers, Notes on Number Theory and Discrete Mathematics 23(1) (2017), 91-98.

[50] Ş. Uygun and S. Yaşamalı, On the bounds for the norms of $r$-circulant matrices with the Jacobsthal and Jacobsthal-Lucas numbers, International Journal of Pure and Applied Mathematics 112(1) (2017), 93-102. https://doi.org/10.12732/ijpam.v112i1.7

[51] C. C. Yalavigi, Properties of Tribonacci numbers, Fibonacci Quart. 10(3) (1972), 231-246.

[52] N. Yilmaz and N. Taskara, Tribonacci and Tribonacci-Lucas numbers via the determinants of special matrices, Appl. Math. Sci. 8(39) (2014), 1947-1955. https://doi.org/10.12988/ams.2014.4270

[53] G. Zielke, Some remarks on matrix norms, condition numbers, and error estimates for linear equations, Linear Algebra Appl. 110 (1988), 29-41. https://doi.org/10.1016/0024-3795(83)90130-1

This is an open access article distributed under the terms of the Creative Commons Attribution License (http://creativecommons.org/licenses/by/4.0/), which permits unrestricted, use, distribution and reproduction in any medium, or format for any purpose, even commercially provided the work is properly cited. 